\title{
What behaviour change techniques are associated with effectiveness of weight management interventions? A systematic review of systematic reviews
}

\author{
C.M. McGirr ${ }^{1}$, S.U. Dombrowski ${ }^{2}$, V.A. Holmes ${ }^{1}$ and M.C. McKinley ${ }^{1}$ \\ ${ }^{1}$ Institute for Global Food Security (Centre for Public Health), School of Medicine, Dentistry and Biomedical \\ Sciences, Queen's University Belfast, BT12 6BJ and \\ ${ }^{2}$ Psychology3A107, University of Stirling, Stirling, FK9 4LA
}

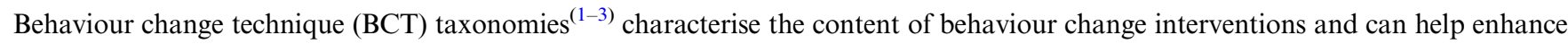
understanding of which intervention components are linked to intervention effectiveness. A number of systematic reviews have examined behavioural interventions for weight management and linked BCTs to explain variation in intervention effectiveness. The aim of this systematic review of systematic reviews is to identify the behaviour change techniques (BCTs) that are linked with effectiveness in weight management interventions for adults.

Electronic databases (MEDLINE, EMBASE, CINAHL, PsycInfo and Cochrane Library) were searched systematically. Inclusion criteria were: i) must be a systematic review of randomised controlled trials or cluster randomised controlled trials of a behavioural intervention for weight management compared to a control condition; ii) include weight as an outcome in the systematic review; iii) interventions included in the systematic review must be coded according to one of three taxonomy's, ${ }^{(1-3)}$; iv) systematic reviews must have linked BCTs or clusters of BCTs to effectiveness, either statistically or narratively.

The search yielded 13,519 articles of which six met the inclusion criteria. Four systematic reviews aimed at assessing BCT use in weight loss interventions for overweight and/or obese adults and two focused on BCTs used in gestational weight management interventions. Authors of the systematic reviews either reported individual BCTs or BCT clusters in their results, (BCT clusters are made up of an extensive list of 93 well defined BCTs. There are 16 BCT clusters in total $\left.{ }^{(3)}\right)$. Four systematic reviews coded BCTs using the CALO-RE taxonomy ${ }^{(2)}$ (includes $40 \mathrm{BCTs}$ ), two of which grouped these into the BCT clusters ${ }^{(3)}$ One systematic review used the original 26 BCT taxonomy ${ }^{(1)}$ and one systematic review used the taxonomy v $1^{(3)}$ (includes 93 BCTs). For comparison between systematic reviews we mapped these BCTs into BCT clusters using taxonomy $\mathrm{vl}^{(3)}$. Two of the six systematic reviews used 13 out of the possible 16 BCT clusters. Two systematic reviews used 11 BCTs clusters, one systematic review used 10 BCT clusters and one systematic review used 9 BCT clusters.

The following BCT clusters were identified in the intervention content in all 6 systematic reviews: 'goals and planning', 'reward and threat', 'feedback and monitoring', 'shaping knowledge', 'comparison of behaviour' and 'repetition and substitution'. These BCT clusters have also been either statistically or narratively linked to effectiveness in weight management interventions.

Retrospective coding of BCTs as part of systematic review of interventions can enhance our understanding of what intervention components are associated with effectiveness. The BCT clusters 'goals and planning' and 'feedback and monitoring' were consistently associated with effectiveness. This work may prove useful for health professionals and researchers to inform development of future weight management interventions.

This work is funded by NIHR and the Harold McAuley Research Scholarship.

1. Abraham C and Michie S. (2008) Health Psychol 27, 379-387.

2. Michie S, Ashford S, Sniehotta FF, et al. (2011) Psychology and Health. 26, 1479-1498.

3. Michie S, Richardsom M, Johnston M, et al. (2013) Ann Behav Med 46, 81-95. 